\title{
ECOEFICIENCIA EN LA UNIVERSIDAD HACIA UN DESARROLLO SOSTENIBLE
}

Yolanda Bustamante Sánchez*

yobusa22@yahoo.es

\section{RESUMEN}

Dada la situación de emergencia ambiental que vivimos se precisa que la universidad tome medidas de ecoeficiencia orientadas al cuidado y equilibrio del medio ambiente a través del uso racional de los recursos, ahorro de energía, agua, papel, generación de residuos y acciones encaminadas al desarrollo sostenible.

Palabras clave: ecoeficiencia, universidad, desarrollo sostenible.

\section{ABSTRACT}

Due to the environmental emergency that we are facing, it is necessary to implement eco efficiency measurements at university oriented to the rational use of resources, water, energy, generation of waste oriented to the sustainable development.

Keywords: Ecoefficiency, University, Sustainable Development.

* Egresada de la Maestría en Administración, mención en Dirección de Recursos Humanos. Diploma en Dirección y Gestión Pública Local - UIM-AECI, Granada, ESPAÑA (La Sostenibilidad Local). Cursos Actualización ITCILO, OIT-TURIN-ITALIA, Gobierno Electrónico- OEA. Docente Investigadora, Coordinadora PIE, Escuela Postgrado UNMSM, Coordinación CLUMA Universidad Peruana Las Américas. Conferencista Internacional 


\section{INTRODUCCIÓN}

Luego de un diagnóstico institucional en la universidad sobre el uso de energía en diferentes ambientes, el uso de focos, fluorescentes prendidos aún durante el día, la instalaciones sanitarias, el uso de agua potable para consumo y regado de jardines, la generación de residuos sólidos, el consumo de papel, el reciclado de productos orgánicos e inorgánicos, se precisa procurar el uso racional de los recursos, ahorrar y optimizar el consumo de papel, energía, agua así como segregar y reciclar residuos sólidos convenientemente, por lo que se propone aplicar medidas de ecoeficiencia institucionales como una alternativa de mejora continua al servicio público, para el desarrollo sostenible.

\section{OBJETIVOS DE LA ECOEFICIENCIA}

- Implementar la mejora continua a través del ahorro y la generación de menos impactos en el ambiente

- Ahorrar recursos materiales, energía (iluminación, equipos ofimáticos, aire acondicionado, bombas de agua)

- Minimizar la generación de residuos

- Esto se traducirá en la mejora de la economía destinada a fines primordiales del desarrollo sostenible

- Adoptar medidas de ecoeficiencia tales como consumo de energía, agua, papel, gastos de combustible en vehículos

- Establecer Buenas Prácticas institucionales a través de la adopción de medidas que permitan el cambio de Comportamiento de los servidores públicos

- Generar cultura de la eficiencia en los colaboradores que pueden trasladar a sus viviendas con significativo impacto en la economía del hogar.

\section{BASE LEGAL}

- Ley 284111- Ley General del Sistema Nacional de Presupuesto

- Ley 29465- Ley de Presupuesto del Sector Público para el Año Fiscal 2010

- Decreto Supremo Nro. 009-2009-MINAMque aprueba Medidas de Ecoeficiencia para el Sector Público.
- Decreto Supremo 063-2007-PCM que aprueba el Reglamento de Organización y Funciones de la Presidencia del Consejo de Ministros, modificado por el Decreto Supremo 057-2008-CM

- Decreto Supremo 009-2009-MINAM

\section{- Resolución 236-2005- OSINERG/CD}

\section{ECOEFICIENCIA Y DESARROLLO SOSTENIBLE}

El término ecoeficiencia fue establecido por el World Business Council for Sustainable Development (WBCSD) en su publicación del año 1992 CHANGING COURSE, con la finalidad de crear más bienes y servicios utilizando menos recursos y creando menos basura y polución encaminado al desarrollo sostenible.

El Desarrollo Sostenible o Sustentable es un concepto que se enmarca bajo un contexto macroeconómico. Las normas y legislaciones en temáticas medioambientales han de incidir en la economía de la institución , sus decisiones estratégicas tal como lo señalan Porter y Varn Der Linde (1995), quienes demostraron que la aplicación de estándares medioambientales encuentra respuesta positiva y altamente competitiva en las empresas, empujando a las instituciones a ser innovadoras. En la misma línea Enticott y Walter (2005) señalan que ha surgido una nueva etapa en la elaboración de políticas medioambientales para las instituciones. Los nuevos conceptos ligados al medio ambiente se vienen tratando por las Naciones Unidas, grupos empresariales y destacadas ONG hace tres décadas.

Los primeros pasos en esta temática los da el Club de Roma en el informe "los límites del crecimiento"(Beyond The Limits To Growth) preparado por Meadows (1972), en el que se cambia la máxima de "crecer o morir" por "crece y muere", ya que se detecta fehacientemente que la tierra tiene límites ambientales. En el mismo año 1972, en Estocolmo, se celebró la primera Cumbre Mundial del Medio Ambiente.

Allí las naciones se pusieron de acuerdo en que era apremiante la necesidad de abordar el problema del deterioro ambiental. Veinte años más tarde, en la Conferencia de las Naciones Unidas sobre Medio ambiente y el Desarrollo, celebrada en Río de Janeiro en 1992, se convino en que la protección del medio ambiente, el desarrollo social y el desarrollo económico eran fundamentales para lograr el desarrollo sostenible. Para 
alcanzar este objetivo se aprobó un programa de alcance mundial titulado Programa 21 o Agenda 21 , con trascendencias nacionales y/o locales y se formuló la Declaración de Río sobre Medio Ambiente y Desarrollo, ambos hechos (programa y declaración) fueron ratificados en la Cumbre de Johannesburgo del 2002.

El desarrollo sostenible ha pasado a ser el principal concepto de los estudios y publicaciones con relación al medio ambiente. Este concepto descansa en tres pilares fundamentales: desarrollo económico, desarrollo social y protección del medio ambiente. El concepto en sí fue esbozado por Gro Harlem Brundtland en su trabajo denominado Nuestro Futuro Común (Our Common Future) más conocido como el Informe Brundtland, el cual señala que el desarrollo sostenible es un desarrollo que satisface las necesidades del presente sin comprometer la capacidad de las futuras generaciones. Este concepto ha sido base de los informes de la ONU y ha tenido aplicabilidad en los distintos gobiernos que hoy implementan políticas de desarrollo sostenible para una mejor calidad de vida a través del cumplimiento de los 18 principios de sostenibilidad:

- Las personas tienen derecho a una vida saludable y productiva en armonía con la naturaleza.

- El desarrollo hoy día no debe socavar las necesidades ambientales y de desarrollo de las generaciones presentes y futuras.

- Los países tienen el derecho soberano de explotar sus propios recursos, pero sin causar daños ambientales más allá de sus fronteras.

- Las naciones deben desarrollar leyes internacionales para ofrecer compensaciones por el daño que las actividades bajo su control causen en áreas más allá de sus fronteras

- Los países deben utilizar un enfoque precautorio para proteger el medio ambiente. Donde existan amenazas de daño serio o irreversible, no debe usarse la incertidumbre científica para posponer la implantación de medidas costo-efectivas para prevenir la degradación ambiental.

- Para lograr el desarrollo sostenible, la protección ambiental debe constituir una parte integral del proceso de desarrollo, y no se puede considerar como un elemento aislado. Es esencial erradicar la pobreza y reducir las disparidades entre los estándares de vida en diferentes partes del mundo para lograr el desarrollo sostenible y satisfacer las necesidades de la mayoría de las personas.

- Las naciones deberán cooperar para conservar, proteger y restaurar la salud e integridad del ecosistema de la Tierra. Los países desarrollados reconocen la responsabilidad que tienen en la búsqueda internacional del desarrollo sostenible en vista de las presiones que sus sociedades imponen al medio ambiente global y de las tecnologías $\mathrm{y}$ recursos financieros que dominan

- Los países deben reducir y eliminar los patrones no sostenibles de producción y consumo, así como promover políticas demográficas apropiadas.

- Las cuestiones ambientales se manejan mejor con la participación de todos los ciudadanos interesados. Las naciones deberán facilitar y fomentar la conciencia y participación pública poniendo la información ambiental a disposición de todos.

- Los países deberán decretar leyes ambientales efectivas, y desarrollar leyes nacionales sobre las obligaciones legales para con las víctimas de la contaminación y otros daños de carácter ambiental. En donde tengan autoridad, los países deberán evaluar el impacto ambiental de actividades propuestas que tengan un potencial y significativo impacto adverso.

- Los países deberán cooperar para promover un sistema económico internacional abierto que lleve al crecimiento económico y desarrollo sostenible de todos los países.

- Las políticas ambientales no deben utilizarse como un medio injustificado de restringir el comercio internacional. En principio, el que contamina debe asumir el costo de la contaminación.

- Las naciones deberán alertarse unas a otras acerca de desastres naturales o actividades que pudieran tener impactos transfronterizos peligrosos.

- El desarrollo sostenible requiere de un mejor entendimiento científico de los 
problemas. Los países deben compartir conocimientos y tecnologías innovadoras para lograr la meta de la sostenibilidad.

- La participación completa de las mujeres es esencial para lograr el desarrollo sostenible. También se necesitan la creatividad, ideales y valor de la juventud y el conocimiento de los grupos indígenas. Los países deben reconocer y apoyar la identidad, cultura e intereses de los grupos indígenas.

- La guerra es inherentemente destructiva del desarrollo sostenible, y las naciones deberán respetar las leyes internacionales que protegen al medio ambiente en tiempos de conflictos armados, y deberán cooperar para que dichas leyes se sigan estableciendo.

- La paz, el desarrollo y la protección ambiental son interdependientes e indivisibles.

Para este fin, una de las herramientas que nos pueden ayudar a evaluar la gestión de los residuos con resultados objetivos, es la medición de la eficiencia desde un punto de vista medioambiental, es decir, la ecoeficiencia que es un término que nace como respuesta a la necesidad de indicadores de productividad y eficiencia en los procesos productivos limpios o verdes, en directo beneficio del desarrollo sostenible.

La Organización para la Cooperación y Desarrollo Económico (OCDE), ha definido la ecoeficiencia como la eficiencia con la cual se usan los recursos ecológicos para satisfacer las necesidades humanas. La Agencia Europea del Medio Ambiente (AEMA) que trata de usar los indicadores de ecoeficiencia para cuantificar el progreso hacia la sostenibilidad a nivel macro, define la eco eficiencia como crear más bienestar con menos recursos naturales y afirma que proviene de desligar el uso de recursos y la contaminación del crecimiento económico.

\section{INDICADORES DE ECOEFICIENCIA}

Curcelle (1998) nos ilustra con cuatro factores que estarían clasificados por un lado a corto y largo plazo y, por otro, en aspectos económicos, medioambientales, sociocultural y técnicos

\begin{tabular}{|c|l|l|}
\hline FACTOR & CORTO PLAZO & LARGO PLAZO \\
\hline \multirow{2}{*}{ ECONÓMICOS } & $\begin{array}{l}\text { Coste de inversión } \\
\text { Coste neto de operación } \\
\text { Coste neto total por tonelada } \\
\text { Coste neto anual } \\
\text { Mercado potencial }\end{array}$ & $\begin{array}{l}\text { Viabilidad a largo plazo de búsqueda } \\
\text { y ordenación de operaciones futuras }\end{array}$ \\
\hline \multirow{3}{*}{ AMBIENTALES } & $\begin{array}{l}\text { Calidad de reconversión de } \\
\text { material } \\
\text { El residuo } \\
\text { La polución } \\
\text { El ruido } \\
\text { El uso de recursos naturales }\end{array}$ & Impacto global: pérdida de biodiver- \\
& Aceptación pública & nes ácidas, paisajismo \\
\hline \multirow{2}{*}{ TÉCNICOS } & Participación & Bivel de salud pública \\
& Empleos & Bienestar, la disponibilidad de re- \\
& cursos naturales (materia y energía) \\
\hline & Escala & Futuro desarrollo potencial \\
\hline
\end{tabular}




\section{VENTAJAS DE LA ECOEFICIENCIA}

Para la institución, la ecoeficiencia es "producir más con menos". Una gestión ecoeficiente de los procesos de producción o de los servicios de una empresa aumenta la competitividad de esta empresa, ya que reduce el despilfarro de recursos mediante la mejora continua, reduce el volumen y toxicidad de residuos generados, reduce el consumo de energía y las emisiones contaminantes, se reducen los riesgos de incumplimiento de las leyes y se favorecen las relaciones con la administración competente.

La ecoeficiencia está dirigida a fin de fomentar una nueva cultura de uso eficiente de los recursos de energía, papel, agua y recursos logísticos que genere un ahorro importante, así como propiciar en los trabajadores que son los principales consumidores de estos servicios, el uso ambientalmente responsable a fin de lograr el menor impacto en el ambiente.

En el espacio universitario, la práctica de la educación ambiental logra un modelo integrador de una educación de calidad enriquecido con practicas ambientales con ecoeficiencia para forjar una cultura de saber resolver problemas ambientales en alianzas, forjar cultura de emprendimientos ambientales, generadores de creatividad, investigación e innovación a través de una educación vivencial, practicas ecoeficientes, saludables, no generando residuos en exceso, ni derrochando sus recursos, su energía.

De este modo, la universidad, como empresa educativa será más competitiva al incorporar una decidida gestión ambiental en sus procesos productivos aprovechando la mayor cantidad de horas de luz natural al día, desconectando los aparatos eléctricos al abandonar las aulas, concientizando el ahorro de agua, reduciendo el uso de papel, promoviendo el reciclaje.

\section{PLAN DE ACCIÓN: MEDIDAS DE ECOEFICIENCIA}

\section{Ahorro de papel y materiales conexos}

a) Impresión de documentos por ambas caras de la hoja de papel que se utilice, con la excepción de aquellos documentos que la Secretaría General de cada institución determine a través una directiva interna específica en la materia. De ser posible se imprimirá a dos páginas por caras. b) Reutilización de papeles en documentos preliminares o de borrador.

c) Utilización con mayor frecuencia de la comunicación electrónica en reemplazo de la escrita, sobre todo en documentos preliminares.

d) Evitar la impresión innecesaria de comunicaciones electrónicas.

e) Utilizar el modo "borrador" en la impresión de los documentos de trabajo que sea indispensable imprimir.

f) Promover el escaneado de todos los documentos recibidos en mesa de partes, a fin que sean compartidos por las dependencias que lo requieran en forma de archivo digital, evitando el fotocopiado sucesivo del mismo documento.

\section{Ahorro de energía}

a) Limpieza periódica de luminarias y ventanas por lo que se llevará un registro de su cumplimiento, asimismo establecer una frecuencia mayor de limpieza de ventanas destinadas para iluminación natural durante el día.

b) Disposición de los puestos de trabajo para un mejor aprovechamiento de la luz y ventilación natural.

c) Optimización de las horas de funcionamiento de oficinas con luz natural.

d) Optimización del uso de ventiladores.

e) Optimización del uso de aire acondicionado de acuerdo a las indicaciones del fabricante, el mantenimiento preventivo y la utilización solo en ambientes que reúnan las condiciones de carga térmica y hermeticidad.

f) Racionalizar la iluminación artificial en horas nocturnas.

g) Apagar los equipos eléctricos y electrónicos cuando no se tenga prevista su inmediata utilización.

h) La Oficina General de Administración de cada institución establecerá mecanismos técnicos y organizacionales para que los equipos se apaguen automáticamente para garantizar el ahorro.

i) Disponer avisos sobre el buen uso de la energía en la institución.

j) Uso de la función "protector de pantalla" estático con fondo negro. 


\section{Medidas cuidado del agua}

a) Control de fugas de agua en las instalaciones internas y servicios sanitarios; la Oficina General de Administración de cada institución dispondrá una evaluación rápida para la identificación de fugas y adoptará las medidas correctivas inmediatas.

b) Disponer avisos sobre el buen uso de los servicios en todos los puntos de agua de la institución.

c) En caso de observar alguna avería en las instalaciones sanitarias así como cualquier forma de pérdida de agua, el personal comunica el hecho, mediante correo electrónico, a la Oficina General de Administración de cada entidad para su inmediata reparación.

d) Riego de jardines en horas de baja intensidad solar.

\section{Segregación y reciclado de residuos sólidos.}

a) Para lograr la ecoeficiencia las entidades del sector público deberán implementar las operaciones de segregación en fuente, a fin de agrupar residuos con características y propiedades similares realizando como mínimo la segregación de:

- Papeles;

- Cartones;

- Plásticos;

- Cartuchos de tinta y tóner de impresión;

- Aluminio y otros metales;

- Vidrios; $y$,

- Otros a consideración de la entidad.

b) La Oficina General de Administración de cada entidad dispondrá las medidas necesarias para la segregación, recolección y almacenamiento temporal interno de los residuos, mediante colocación de contenedores diferenciados

c) Los materiales segregados serán entregados a entidades o empresas recicladoras debidamente registradas ante la autoridad competente, y cuya relación se publicará en el portal institucional del Ministerio del Ambiente, MINAM

\section{MEDIDAS ADICIONALES}

En la institución prever la instalación de domos o tragaluces para que la luz solar entre al edificio. Prever la utilización de puertas transparentes o traslúcidas en las salas ubicadas en las oficinas centrales para permitir que tengan acceso a la luz natural. Las apariciones de oficina pueden ser de vidrio claro, vidrio ahumado o bloques de vidrio. Los pisos deberán de utilizar lozas de color claro para mejorar la reflectividad y reducir la cantidad de luz absorbido por el piso. Procurar que las paredes y los techos de las salas deberían ser de color claro (blanco ) y presentar un acabado liso y mate para reducir la cantidad de luz absorbida por estas superficies, mejorar el aprovechamiento de la luz natural disponible y ahorrar energía al reducir la cantidad de luz artificial necesaria para lograr el grado de iluminación

Se recomienda instalar ventiladores de techo en las oficinas que no cuentan con aire acondicionado, evitar el uso de aire acondicionado

\section{BIBLIOGRAFÍA}

Ecoeficiencia en el Sector Público - Resolución de Secretaría General 009-2010 (16 agosto 2010)

Medidas de Ecoeficiencia para el Sector PúblicoDecreto Supremo 002-2009-MINAM

L. Hunter Lovins. Rethinking Production EcoEfficiency - World Business Council For Sustainable Development

Schmidheiny Stephan. World Business Council for Sustainable Development. Eco-Efficiency Creating Some Value With Less Impact

IBM- The Emergence Of The Eco-Efficient Economy. Ibm Global Business Services

Charpentier Silvia e Hidalgo Jessica. Las Políticas ambientales en el Perú. Octubre 1999

Fundación FORUM AMBIENTAL. Guía para la ecoeficiencia. 


\section{ANEXOS}

\section{ENCUESTA}

A través de la siguiente encuesta se aprecia la realidad de las áreas de la universidad

1. ¿Ha habido alguna iniciativa para controlar el consumo de energía en las horas punta orientada a reducir la tarifa?

2. ¿Los focos, fluorescentes permanecen encendidos durante el día?

3. ¿Ha habido una inspección del estado de las instalaciones sanitarias interiores en los últimos meses?

4. ¿Hay un registro estadístico de la facturación de agua potable?
5. ¿Conoce cuánto residuos sólidos orgánicos e inorgánicos se generan en su facultad?

6. ¿Dispone de un programa de clasificación de residuos sólidos en la Facultad?

7. ¿Existe una actividad de Capacitación / concientización de Buenas Prácticas Ambientales en su Facultad?

8. ¿Conoce las medidas de eco eficiencia en su Facultad?

9. ¿En el área administrativa se imprime el papel en ambas caras?

10. ¿Se aprovecha la luz natural de las aulas y oficinas a fin de hacer ahorro de energía eléctrica en la Facultad? 